\title{
mHealth Interventions for Self-management of Hypertension: Framework and Systematic Review on Engagement, Interactivity, and Tailoring
}

Weidan $\mathrm{Cao}^{1}$, PhD; M Wesley Milks², MD; Xiaofu Liu ${ }^{1}, \mathrm{MSc}$; Megan E Gregory ${ }^{1,3}, \mathrm{PhD}$; Daniel Addison ${ }^{2}$, MD; Ping Zhang ${ }^{1}$, PhD; Lang $\mathrm{Li}^{1}$, PhD

\footnotetext{
${ }^{1}$ Department of Biomedical Informatics, The Ohio State University, Columbus, OH, United States

${ }^{2}$ Division of Cardiovascular Medicine, The Ohio State University College of Medicine, Columbus, OH, United States

${ }^{3}$ The Center for the Advancement of Team Science, Analytics, and Systems Thinking (CATALYST), The Ohio State University College of Medicine, Columbus, $\mathrm{OH}$, United States
}

\section{Corresponding Author:}

Lang $\mathrm{Li}, \mathrm{PhD}$

Department of Biomedical Informatics

The Ohio State University

250 Lincoln Tower, 1800 Cannon Drive

Columbus, OH, 43210

United States

Phone: 16142924778

Email: lang.li@osumc.edu

\section{Abstract}

Background: Engagement is essential for the effectiveness of digital behavior change interventions. Existing systematic reviews examining hypertension self-management interventions via mobile apps have primarily focused on intervention efficacy and app usability. Engagement in the prevention or management of hypertension is largely unknown.

Objective: This systematic review explores the definition and role of engagement in hypertension-focused mobile health (mHealth) interventions, as well as how determinants of engagement (ie, tailoring and interactivity) have been implemented.

Methods: A systematic review of mobile app interventions for hypertension self-management targeting adults, published from 2013 to 2020, was conducted. A total of 21 studies were included in this systematic review.

Results: The engagement was defined or operationalized as a microlevel concept, operationalized as interaction with the interventions (ie, frequency of engagement, time or duration of engagement with the program, and intensity of engagement). For all 3 studies that tested the relationship, increased engagement was associated with better biomedical outcomes (eg, blood pressure change). Interactivity was limited in digital behavior change interventions, as only 7 studies provided 2-way communication between users and a health care professional, and 9 studies provided 1-way communication in possible critical conditions; that is, when abnormal blood pressure values were recorded, users or health care professionals were notified. The tailoring of interventions varied at different aspects, from the tailoring of intervention content (including goals, patient education, advice and feedback from health professionals, reminders, and motivational messages) to the tailoring of intervention dose and communication mode. Tailoring was carried out in a number of ways, considering patient characteristics such as goals, preferences, disease characteristics (eg, hypertension stage and medication list), disease self-management experience levels, medication adherence rate, and values and beliefs.

Conclusions: Available studies support the importance of engagement in intervention effectiveness as well as the essential roles of patient factors in tailoring, interactivity, and engagement. A patient-centered engagement framework for hypertension self-management using mHealth technology is proposed here, with the intent of facilitating intervention design and disease self-management using mHealth technology.

(JMIR Mhealth Uhealth 2022;10(3):e29415) doi: 10.2196/29415 


\section{KEYWORDS}

mHealth; mobile app; digital behavior change; interventions; systematic review; hypertension; engagement; interactivity; tailoring; mobile phone

\section{Introduction}

\section{Background}

Hypertension is an impactful risk factor for heart disease and stroke, both of which are leading causes of death in the United States [1]. Approximately $45 \%$ of American adults have a diagnosis of hypertension, and only $24 \%$ of those with hypertension have their condition under optimal control [1]. Effective treatment of hypertension requires patients to work with their health care providers and follow self-management guidelines, particularly relating to medication adherence.

Mobile health (mHealth) is defined as the use of mobile technologies (eg, smartphones) to provide medical and public health practice [2]. mHealth interventions used for disease self-management belong to digital behavior change interventions, defined as those involving digital technologies (eg, mHealth apps) to promote or support behavior change for improved health and self-management of chronic disease [3,4] for better health, which have been used to facilitate hypertension self-management. Potential benefits of mHealth interventions for disease self-management include (1) increasing medication adherence [5], (2) increasing knowledge, (3) empowering patients for self-care, (4) providing personalized self-care recommendations, and (5) facilitating patient-care provider communication and decision-making [6,7]. Hundreds of mHealth apps, often with features such as educational resources and monitoring reminders, have previously been developed to support hypertension self-management [8], and studies have demonstrated the effectiveness of using apps in blood pressure (BP) control and self-management behavior change such as medication adherence $[6,9,10]$.

Existing systematic reviews examining the mHealth interventions for hypertension self-management or digital behavior change interventions using mHealth in the context of hypertension have focused on intervention efficacy and app usability $[6,9,11]$. However, studies examining engagement in the context of hypertension are limited. Engagement can be defined from a microlevel perspective (ie, engagement with digital behavior change interventions only, such as intervention use, or the subjective experience characterized by attention, affect, and interest) or from a macrolevel perspective (ie, engagement with the broader landscape of behavior change, such as medication adherence) [3,4]. Macrolevel engagement can be the result of the microlevel of engagement. For instance, engagement (eg, frequency, amount, and duration) with interventions can lead to behavior engagement or change (eg, medication adherence). Engagement is essential for the effectiveness of interventions involving digital technology $[3,12,13]$, whereas lack of engagement with mHealth interventions would be expected to be associated with a lower rate of intervention success [14]. Subsequently, understanding the determinants of engagement can help design effective interventions. Theoretical frameworks $[15,16]$ have proposed specific strategies to engage patients using mobile apps. Such strategies include but are not limited to providing educational information, reminding or alerting users, recording and tracking health information, providing guidance based on information entered by the user, enabling communication with clinicians, providing support through social networks, and supporting behavior change through rewards $[15,16]$.

Although prior work has examined engagement in the context of chronic conditions [17,18], it has largely focused only on the microlevel of engagement $[17,18]$, thus leaving the macrolevel predominantly unexamined. Intervention effectiveness measured by app use alone cannot be taken as a valid indicator of engagement because use metrics (microlevel engagement) do not indicate offline engagement indicators, and microlevel disengagement with the intervention or technology does not necessarily preclude macrolevel engagement (eg, users may take medications adherently but do not use the app to track medication taking behaviors) [4].

Therefore, it is of vital importance to examine both types of engagement as well as their determinants in mHealth behavior change interventions [19]. According to the motivational technology model [20], customization (ie, tailoring) and interactivity are the two key determinants of engagement. Tailoring refers to the extent to which users can customize the mHealth intervention to meet their needs [21]. For instance, an app may tailor the educational content delivered, messages, alerts, and reminders, and displays to users' specific needs and preferences; a patient on medication to manage severe hypertension likely requires different features and messaging than a patient who is managing mild hypertension through lifestyle modifications. Interactivity refers to the opportunities that the mHealth intervention affords for users to communicate with others, especially health care professionals [21]. For example, apps that have coaching from a trained professional tend to be more interactive. Along these lines, systematic reviews focusing on mHealth disease self-management interventions found that effective interventions integrated features of interactive communication [10] and tailored messages [22]. A systematic review of nutrition apps found that tailoring the apps to the needs of specific user groups can be beneficial in increasing engagement [23]. In addition, interventions can be more engaging if they are designed to be tailored to participants' health beliefs and needs. For instance, a systematic review of studies on health beliefs and medication adherence in patients with hypertension found that medication adherence was related to health beliefs that vary within and across countries, such as disease severity and susceptibility, medication necessity, or efficacy [24]. This implies that medication adherence interventions need to consider individual health beliefs about hypertension and BP medications [24]. 


\section{Objective}

Therefore, based on the importance of engagement, the research gap, the determinants of engagement, and the strategies to engage users, the following research questions were proposed:

1. What engagement strategies have been used in digital behavior change interventions for hypertension self-management?

2. How has engagement been defined or presented in the literature on digital behavior change interventions for hypertension self-management?

3. How has interactivity been implemented in digital behavior change interventions for hypertension self-management?

4. How has tailoring been implemented in digital behavior change interventions for hypertension self-management?

\section{Methods}

\section{Overview}

A systematic review of the literature was conducted to identify extant interventions and to investigate their key characteristics. Subsequently, content analyses of the studies were conducted for a deeper understanding of how engagement and its determinants were implemented in the interventions.

\section{Search Strategy}

The search focused on the identification of studies relating to mHealth interventions for hypertension self-management conducted worldwide. The search was conducted on the following databases: PubMed, PsycINFO, Embase, Communication and Mass Media Complete, CINAHL, MEDLINE, and MEDLINE Full Text. All articles indexed as of June 2020 were searched. A combination of search terms was used, including hypertension, hypertensive, hypertensives, or blood pressure (for the disease type); self-management, self management, self-care, self care, management, coaching, control, monitor, adhere, or adherence (for disease management); mHealth, m-health, mobile, app, apps, application, applications, smart phone, smartphone, technology (for mHealth); intervention, trial, program, programme, experiment, pilot, study, effect, experience, or experiences (for intervention). Please refer to Multimedia Appendix 1 for the search strategy and the corresponding justifications.

To ensure the comprehensiveness of the search, we also scanned relevant journals (eg, JMIR mHealth and uHealth) and the reference lists of review articles about these interventions.

\section{Study Selection}

Included studies were those conducted among adults (aged $\geq 18$ years), involving a mobile app to facilitate hypertension self-management, and with the aim of testing app or system experience. If an app was designed specifically for hypertension management for patients with hypertension, then the study was included (eg, the study by Kang and Park [25]). If an app was used for BP reduction or hypertension and another health condition (eg, weight management), the study was included (eg, the study by Mao et al [26]). If an app was designed for a specific purpose (eg, medication adherence) and could be applied to different health conditions or diseases, and was applied to patients with hypertension in the study, the study was included (eg, the study by Morawski et al [27]). If an app was used for $>1$ disease (eg, for both diabetes and hypertension) and if patients with hypertension or patients with both conditions were included as participants in the study, then the study was included [14]. If an app was used among patients with other diseases or conditions (eg, kidney transplant) and managing hypertension is crucial for that disease or condition, then the study was included (eg, the study by McGillicuddy et al [28]).

The exclusion criteria included articles that met one or more of the following characteristics: (1) use of apps for the purposes of disease screening or disease detection; (2) focus only on app design and development, without reporting any participants' app use experience; (3) primarily designed for healthcare providers or that reported professionals' user experience but did not focus on patients' user experience; (4) study of children; (5) based solely on non-smart phones, on the internet, or on text messages; (6) not written in English, and (7) contained only an abstract, without full publication. Covidence [29] was used to manage the review process.

A total of 442 records were imported to Covidence, and 223 duplicates were removed. After title and abstract screening, among the remaining 219 articles, 138 (63\%) were removed based on not meeting the inclusion criteria. The remaining $37 \%$ $(81 / 219)$ articles were screened, and $69 \%(56 / 81)$ of them not meeting the inclusion criteria were removed at this stage. A further review of the remaining 31\% (25/81) of the articles indicated that $16 \%(4 / 25)$ of the articles [30-33] were based on the same app or intervention system. The study by Bengtsson et al [30] was not included in this review because it focused more on system development. The other study [33] was not included in this review because the patient participants were a subgroup of the patient participants in another study [32]. Furthermore, the studies by McGillicuddy et al $[28,34]$ were based on the same system, and one of these studies [34] was not included because the other study [28] built on the 3-month randomized control trial conducted by McGillicuddy et al [34] and was a follow-up of that study. In addition, studies by Persell et al $[35,36]$ were based on the same mobile app, so one of the studies [35] was removed because it focused more on the design of the app. Furthermore, the studies by Moore et al [37] and Thies et al [14] were based on the same app, and the studies by Chandler et al [38] and Davidson et al [39] were based on the same smartphone medication adherence stops hypertension program. For those studies that used the same system or app, if the participants, goals or outcomes, or methods (eg, surveys or interviews) were different, they were included in the review. For instance, although Moore et al [37] and Thies et al [14] used the same system, Moore et al [37] provided positive evidence that the system was effective in hypertension management, whereas Thies et al [14] analyzed the reasons why their intervention failed by using participant interviews. Including both articles would allow a full understanding of the effectiveness of the app or system. Therefore, the final sample size of the review is 21 . A total of 2 authors (ie, WC and XL) worked independently during the screening and selection process first and then compared their results. Discrepancies were resolved through one round of discussion. Figure 1 presents the 
PRISMA (Preferred Reporting Items for Systematic Reviews and Meta-Analyses) flowchart.

Figure 1. PRISMA (Preferred Reporting Items for Systematic Reviews and Meta-Analyses) flowchart.

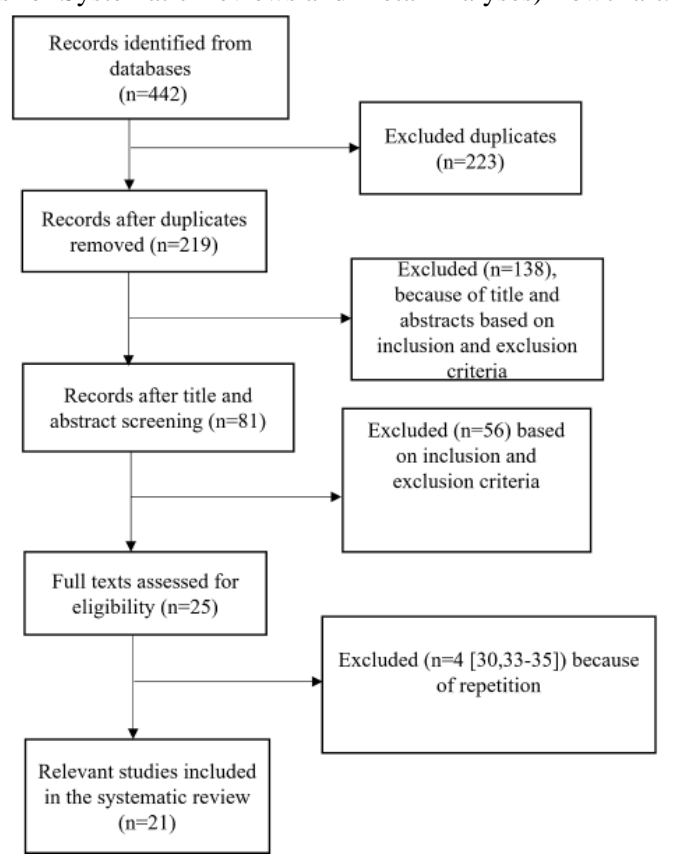

\section{Quality Assessment and Data Analysis}

For quality assessment, 2 coders (WC and XL) independently evaluated the quality of the included studies using four sets of risk of bias evaluation tools: Cochrane Collaboration's Risk of Bias Tool for randomized control trials [40], 2 tools from the National Institutes of Health for observational studies and pre-post studies without a control group [41], and the critical appraisal skills program for qualitative studies [42]. For the study [43] that focused on both qualitative and quantitative data, we used two sets of criteria (ie, pre-post study with no control and qualitative study) to evaluate the risk of bias. These tools were chosen because they have been applied to previously published systematic reviews [9]. Disagreements between the coders were resolved after rounds of discussion and consultation with a third researcher (PZ). The results of the evaluation are in Multimedia Appendix 2 [27,36-39,44-47], Multimedia Appendix 3 [26,28,48,49], Multimedia Appendix 4 [25,31,43,50-52], and Multimedia Appendix 5 [14,32,43].

For the systematic review, 2 coders (WC and XL) independently coded the 21 studies. After independent coding, discrepancies were identified and resolved through multiple rounds of discussion and recoding. Through this iterative process, full agreement was reached for all variables of the systematic review. For coding, the investigators relied on the reporting in the article and referred to related articles listed in the references when applicable (eg, when coding an app that was published in multiple papers). If studies included both patients' and providers' perspectives, only the patients' perspectives were coded. In the event that the article presented no relevant information or the description was general or vague, we coded it as unknown.

\section{Results}

\section{Study Characteristics}

There were 21 studies included in the final analysis, with publication years ranging from 2013 to 2020. Most studies $(14 / 21,67 \%)$ were conducted in the United States. Moreover, of the 21 studies, $2(10 \%)$ were conducted in China, and $2(10 \%)$ other studies were conducted in Sweden. The remaining $14 \%$ $(3 / 21)$ of the studies were conducted in Canada, South Korea, and Spain. The sample size ranged from 17 to 5115 participants, with mean age of 42.44 to 60 years.

The interventions were either developed for general audiences (eg, those who were overweight (BMI $>25 \mathrm{~kg} / \mathrm{m}^{2}$; [26]), for specific audiences with hypertension (eg, patients with poorly controlled hypertension [27], or for patients with diabetes, hypertension, or both [14]. Of the 21 studies, 9 (43\%) were randomized control studies [27,36-39,44-47], 4 (19\%) were observational studies $[26,28,48,49], 6(29 \%)$ were pre-post studies without a control group [25,31,43,50-52], and $2(10 \%)$ were qualitative studies [14,32]. Engagement or self-management behaviors (eg, medication adherence) were not the focus or outcome of longitudinal studies (eg, the study by McGillicuddy et al [28]). In terms of the intervention content, most studies [14,25,27,28,31,32,36-39,43-45,47,48,51] involved medication tracking or medication adherence.

In addition, $24 \%(5 / 21)$ of the studies used a theoretical framework in their interventions. Specifically, some studies $[28,38,39]$ used the self-determination theory. Other theories, including the health belief model and technology acceptance model [43], and the technology-supported apprenticeship model [37] were also applied. No other studies reported any theoretical models. Table 1 presents a summary of the intervention characteristics. 
Table 1. Intervention characteristics.

\begin{tabular}{|c|c|c|c|c|c|c|}
\hline Study & Country & Sample size & $\begin{array}{l}\text { Participants' demographic and } \\
\text { hypertension characteristics }\end{array}$ & Duration & Outcomes & Theory \\
\hline $\begin{array}{l}\text { Bengtsson et al } \\
{[31]}\end{array}$ & Sweden & 50 & $\begin{array}{l}\text { Mean age } 59.5 \text { years; being } \\
\text { currently treated for hyperten- } \\
\text { sion; mean } \mathrm{SBP}^{\mathrm{a}} 142, \text { mean } \\
\mathrm{DBP}^{\mathrm{b}} 84\end{array}$ & 56 days or 8 weeks & $\begin{array}{l}\text { SBP and DBP, identification of sub- } \\
\text { sets or classes of patients who dif- } \\
\text { fered from each other with respect to } \\
\text { level of } \mathrm{BP}^{\mathrm{c}} \text { at baseline }\end{array}$ & No \\
\hline $\begin{array}{l}\text { Chandler et al } \\
{[38]}\end{array}$ & $\begin{array}{l}\text { United } \\
\text { States }\end{array}$ & $\begin{array}{l}54\left(\mathrm{IG}^{\mathrm{d}}=26 ;\right. \\
\text { enhanced } \\
\text { standard } \\
\text { care }=28)\end{array}$ & $\begin{array}{l}\text { I mean age } 44.4 \text { years; en- } \\
\text { hanced standard group mean } \\
\text { age } 46.8 \text { years; Hispanic or } \\
\text { Latino participants; diagnosed } \\
\text { with and prescribed medica- } \\
\text { tion(s) for essential hyperten- } \\
\text { sion; uncontrolled hypertension }\end{array}$ & 9 months & $\begin{array}{l}\mathrm{PO}^{\mathrm{e}} \text { : change in resting SBP from } \\
\text { baseline to the 6-mo time point; } \mathrm{SO}^{\mathrm{f}} \text { : } \\
\text { resting DBP and } \mathrm{MA}^{\mathrm{g}}\end{array}$ & $\begin{array}{l}\text { Self-deter- } \\
\text { mination } \\
\text { theory }\end{array}$ \\
\hline $\begin{array}{l}\text { Ciemins et al } \\
{[49]}\end{array}$ & $\begin{array}{l}\text { United } \\
\text { States }\end{array}$ & $\begin{array}{l}\mathrm{IG}=131 ; \\
\mathrm{CG}^{\mathrm{h}}=353\end{array}$ & $\begin{array}{l}\text { Mean age } 60 \text { years; patients } \\
\text { with newly diagnosed or persis- } \\
\text { tently uncontrolled } \mathrm{BP}^{\mathrm{h}} \\
(\mathrm{ie} \geq 140 / 90 \mathrm{~mm} \mathrm{Hg} \text { ) }\end{array}$ & 32 weeks & $\begin{array}{l}\text { Patients' compliance with study pro- } \\
\text { tocol of taking } 3 \text { BPs per week }\end{array}$ & No \\
\hline $\begin{array}{l}\text { Davidson et al } \\
\text { [39] }\end{array}$ & $\begin{array}{l}\text { United } \\
\text { States }\end{array}$ & $\begin{array}{l}38(\mathrm{IG}=18 \\
\mathrm{CG}=20)\end{array}$ & $\begin{array}{l}\text { I mean age } 47.5 \text { years; } 47 \% \\
\text { (18/38) African Americans and } \\
53 \% \text { (20/38) Hispanics; uncon- } \\
\text { trolled hypertension }\end{array}$ & 6 months & $\begin{array}{l}\text { Changes in clinic SBP and changes } \\
\text { in clinic DBP; changes in SBP con- } \\
\text { trol; changes in DBP control; recruit- } \\
\text { ment and retention rates; MA; BP } \\
\text { adherence }\end{array}$ & $\begin{array}{l}\text { Self-deter- } \\
\text { mination } \\
\text { theory }\end{array}$ \\
\hline Duan et al [43] & China & 143 & $\begin{array}{l}\text { Aged }>18 \text { years, hypertension } \\
\text { diagnosis with no other serious } \\
\text { complications }\end{array}$ & 2 months & $\begin{array}{l}\text { Patient compliance with hypertension } \\
\text { self-management }\end{array}$ & $\begin{array}{l}\text { Health be- } \\
\text { lief model } \\
\text { and the } \\
\text { technology } \\
\text { acceptance } \\
\text { model }\end{array}$ \\
\hline Gong et al [44] & China & $\begin{array}{l}480 \\
(\mathrm{IG}=225 \\
\mathrm{CG}=218)\end{array}$ & $\begin{array}{l}\text { I mean age } 58.2 \text { years; } C \text { mean } \\
\text { age } 59.27 \text { years; patients aged } \\
18 \text { to } 79 \text { years diagnosed with } \\
\text { primary hypertension }\end{array}$ & 6 months & $\begin{array}{l}\text { PO: SBP and DBP changes in pa- } \\
\text { tients; change in percentage of partic- } \\
\text { ipants in the } 2 \text { groups with controlled } \\
\text { BP. SO: MA }\end{array}$ & No \\
\hline $\begin{array}{l}\text { Hallberg et al } \\
\text { [32] }\end{array}$ & Sweden & 49 & $\begin{array}{l}\text { Female median age } 58 \text { years; } \\
\text { male median age } 62.5 \text { years; } \\
\text { female years with hypertension } \\
\text { median } 8 \text {; male years with hy- } \\
\text { pertension median } 6.6\end{array}$ & 8 weeks & $\begin{array}{l}\text { Understanding of the interplay be- } \\
\text { tween BP and daily life; motivation } \\
\text { to follow treatment }\end{array}$ & No \\
\hline $\begin{array}{l}\text { Kang and Park } \\
{[25]}\end{array}$ & $\begin{array}{l}\text { South } \\
\text { Korea }\end{array}$ & 38 & $\begin{array}{l}\text { Mean age } 56 \text { years; patients } \\
\text { with hypertension who take } \\
\text { antihypertensive medications } \\
\text { (taking } 1 \text { or more antihyperten- } \\
\text { sive drugs) }\end{array}$ & 4 weeks & $\begin{array}{l}\text { MA, perceived usefulness, user satis- } \\
\text { faction }\end{array}$ & No \\
\hline $\begin{array}{l}\text { Kaplan et al } \\
{[48]}\end{array}$ & $\begin{array}{l}\text { United } \\
\text { States }\end{array}$ & 5115 & $\begin{array}{l}\text { Mean age } 49 \text { years; mean } \\
\text { SBP130 } \mathrm{mm} \mathrm{Hg} \text {; participants } \\
\text { who recorded } \geq 2 \mathrm{BP} \text { measure- } \\
\text { ments were included in the } \\
\text { study }\end{array}$ & 22 weeks & $\begin{array}{l}\text { Use pattern (engagement), efficacy } \\
\text { of the app in BP reduction }\end{array}$ & No \\
\hline Mao et al [26] & $\begin{array}{l}\text { United } \\
\text { States }\end{array}$ & $\begin{array}{l}\mathrm{IG}=763 \\
\mathrm{CG}=73\end{array}$ & $\begin{array}{l}\text { I mean age } 44.78 \text { years; over- } \\
\text { weight (defined as BMI }>25 \\
\mathrm{~kg} / \mathrm{m}^{2} ; 14.3 \%(109 / 763) \text { partic- } \\
\text { ipants self-reported hyperten- } \\
\text { sion }\end{array}$ & $\begin{array}{l}\text { First } 4 \text { months of } \\
\text { intensive active } \\
\text { coaching and } 8 \\
\text { months of mainte- } \\
\text { nance coaching }\end{array}$ & $\begin{array}{l}\text { PO: weight loss at } 4 \text { months as de- } \\
\text { fined by percent change in total body } \\
\text { weight. SO: change in SBP after } 4 \\
\text { months of intensive health coaching, } \\
\text { as well as the change in number of } \\
\text { participants in each hypertensive cat- } \\
\text { egory from the beginning of enroll- } \\
\text { ment to after } 4 \text { months of coaching }\end{array}$ & No \\
\hline $\begin{array}{l}\text { Márquez Contr- } \\
\text { eras et al [45] }\end{array}$ & Spain & $\begin{array}{l}148(\mathrm{IG}=73 ; \\
\mathrm{CG}=75)\end{array}$ & $\begin{array}{l}\text { Mean age } 57.5 \text { years; patients } \\
\text { with mild to moderate arterial } \\
\text { hypertension }\end{array}$ & $\begin{array}{l}18 \text { months (with an } \\
\text { inclusion period of } \\
6 \text { months and a fol- } \\
\text { low-up of } 12 \\
\text { months) }\end{array}$ & $\begin{array}{l}\text { Pharmacological adherence and con- } \\
\text { trol of BP in patients with mild to } \\
\text { moderate arterial hypertension }\end{array}$ & No \\
\hline
\end{tabular}




\begin{tabular}{|c|c|c|c|c|c|c|}
\hline Study & Country & Sample size & $\begin{array}{l}\text { Participants' demographic and } \\
\text { hypertension characteristics }\end{array}$ & Duration & Outcomes & Theory \\
\hline $\begin{array}{l}\text { McGillicuddy } \\
\text { et al [28] }\end{array}$ & $\begin{array}{l}\text { United } \\
\text { States }\end{array}$ & $\mathrm{IG}=8 ; \mathrm{CG}=9$ & $\begin{array}{l}\text { I mean age } 42.44 \text { years, } C \text { mean } \\
\text { age } 57.89 \text { years; renal trans- } \\
\text { plant recipients with hyperten- } \\
\text { sion with documented medica- } \\
\text { tion nonadherence }\end{array}$ & $\begin{array}{l}12 \text { months after the } \\
\text { completion of a } 3- \\
\text { month randomized } \\
\text { control trial }\end{array}$ & SBP & $\begin{array}{l}\text { Self-deter- } \\
\text { mination } \\
\text { theory }\end{array}$ \\
\hline Moore et al [37] & $\begin{array}{l}\text { United } \\
\text { States }\end{array}$ & $\begin{array}{l}42(\mathrm{IG}=20 \\
\mathrm{CG}=22)\end{array}$ & $\begin{array}{l}\text { Mean age } 50.0 \text { years; patients } \\
\text { with essential hypertension } \\
\text { (average } \mathrm{BP} \geq 140 / 90 \text { and } \\
\leq 180 / 120 \text { ) who were taking } 0 \\
\text { or } 1 \text { medications }\end{array}$ & 12 weeks & $\begin{array}{l}\text { PO: absolute decrease in SBP and } \\
\text { DBP and the number of participants } \\
\text { who reached the BP goal of } \leq 130 / 80 \\
\mathrm{~mm} \mathrm{Hg} \text {. SO: the number of partici- } \\
\text { pants who reached the BP goal of } \\
\leq 140 / 90 \mathrm{~mm} \mathrm{Hg} \text {, the number of par- } \\
\text { ticipants who achieved }>10 \mathrm{~mm} \mathrm{Hg} \\
\text { decreases in SBP and }>5 \mathrm{~mm} \mathrm{Hg} \mathrm{de-} \\
\text { creases in DBP, the change in medica- } \\
\text { tion load, the absolute decrease in } \\
\text { weight, the number of patients who } \\
\text { lost at least } 2.3 \mathrm{~kg} \text {, hypertension } \\
\text { knowledge, satisfaction in care, and } \\
\text { the amount of clinician time required } \\
\text { in the care }\end{array}$ & $\begin{array}{l}\text { A technolo- } \\
\text { gy-support- } \\
\text { ed appren- } \\
\text { ticeship }\end{array}$ \\
\hline $\begin{array}{l}\text { Morawski et al } \\
{[27]}\end{array}$ & $\begin{array}{l}\text { United } \\
\text { States }\end{array}$ & $\begin{array}{l}\mathrm{IG}=209 \\
\mathrm{CG}=202\end{array}$ & $\begin{array}{l}\text { I mean age } 51.7 \text { years; } C \text { age } \\
\text { mean=52.4 years; patients with } \\
\text { poorly controlled hypertension }\end{array}$ & 12 weeks & $\begin{array}{l}\text { PO: change in self-reported MA and } \\
\text { SBP. SO: whether participants had } \\
\text { well-controlled BP, defined as 140/90 } \\
\text { mm Hg or less }\end{array}$ & No \\
\hline $\begin{array}{l}\text { Ovbiagele et al } \\
{[47]}\end{array}$ & $\begin{array}{l}\text { United } \\
\text { States }\end{array}$ & $\begin{array}{l}24(\mathrm{IG}=8 \\
\mathrm{CG}=16)\end{array}$ & $\begin{array}{l}\text { Patients with hypertension after } \\
\text { stroke }\end{array}$ & 3 months & $\begin{array}{l}\text { SBP; emergency department use re- } \\
\text { duction }\end{array}$ & No \\
\hline Patel et al [51] & $\begin{array}{l}\text { United } \\
\text { States }\end{array}$ & 48 & $\begin{array}{l}\text { Mean age } 53 \text { years; African } \\
\text { American } 96 \%(46 / 48) \text {; estab- } \\
\text { lished essential hypertension; } \\
\text { prescribed at least two antihy- } \\
\text { pertensive medications }\end{array}$ & $\begin{array}{l}\text { 12-week activation } \\
\text { (intervention) } \\
\text { phase }\end{array}$ & $\begin{array}{l}\text { PO: MA; SO: MA, level of BP con- } \\
\text { trol by clinic measures, pill phone } \\
\text { use, patient satisfaction, hypertension } \\
\text { medication number and changes dur- } \\
\text { ing the study period, office visits, } \\
\text { emergency room visits, and hospital- } \\
\text { ization }\end{array}$ & No \\
\hline $\begin{array}{l}\text { Persell et al } \\
{[36]}\end{array}$ & $\begin{array}{l}\text { United } \\
\text { States }\end{array}$ & $\begin{array}{l}\mathrm{IG}=144 \\
\mathrm{CG}=153\end{array}$ & $\begin{array}{l}\text { I mean age } 59.6 \text { years; } \mathrm{C} \text { mean } \\
\text { age } 58.3 \text { years; adults with un- } \\
\text { controlled hypertension (de- } \\
\text { fined as at least } 145 \mathrm{~mm} \mathrm{Hg} \\
\text { systolic or } 95 \mathrm{~mm} \mathrm{Hg} \text { diastolic) }\end{array}$ & 6 months & $\begin{array}{l}\text { PO: SBP at } 6 \text { months. SO: self-report- } \\
\text { ed antihypertensive MA, home moni- } \\
\text { toring and self-management practices, } \\
\text { measures of self-efficacy associated } \\
\text { with BP, weight, and health behaviors }\end{array}$ & No \\
\hline $\begin{array}{l}\text { Petrella et al } \\
{[46]}\end{array}$ & Canada & $\begin{array}{l}\mathrm{IG}=67 \\
\mathrm{CG}=60\end{array}$ & $\begin{array}{l}\text { I mean age } 56.7 \text { years; } C \text { mean } \\
\text { age } 59.1 \text { years; participants } \\
\text { with at least two metabolic } \\
\text { syndrome risk factors }\end{array}$ & $\begin{array}{l}52 \text { weeks including } \\
12 \text { weeks of inter- } \\
\text { vention }\end{array}$ & $\begin{array}{l}\text { PO: SBP and other cardiometabolic } \\
\text { risk factors. SO: DBP, waist circum- } \\
\text { ference, lipids (with the exception of } \\
\text { high-density lipoprotein cholesterol, } \\
\text { which was expected to increase) and } \\
\text { markers for blood glucose and inflam- } \\
\text { mation }\end{array}$ & No \\
\hline Thies et al [14] & $\begin{array}{l}\text { United } \\
\text { States }\end{array}$ & $\begin{array}{l}15 \text { out of } 22 \\
\text { downloaded } \\
\text { the app }\end{array}$ & $\begin{array}{l}\text { Mean age } 50 \text { years ( } 22 \text { partici- } \\
\text { pants); } 27 \%(6 / 22) \text { of the pa- } \\
\text { tients with diabetes, } 18 \%(4 / 22) \\
\text { with hypertension, and } 55 \% \\
(12 / 22) \text { with both }\end{array}$ & $\begin{array}{l}\text { Trial suspended, } \\
\text { owing to low en- } \\
\text { rollment and incon- } \\
\text { sistent use of the } \\
\text { app }\end{array}$ & $\begin{array}{l}\text { The original aim of this study was to } \\
\text { evaluate the effectiveness of a com- } \\
\text { mercial mHealth app in improving } \\
\text { clinical outcomes for adult patients } \\
\text { with uncontrolled diabetes or hyper- } \\
\text { tension, or both. Because of low en- } \\
\text { rollment and low app use, the project } \\
\text { aim was changed to understanding } \\
\text { why the trial was unsuccessful }\end{array}$ & No \\
\hline $\begin{array}{l}\text { Toro-Ramos et } \\
\text { al [50] }\end{array}$ & $\begin{array}{l}\text { United } \\
\text { States }\end{array}$ & 50 & $\begin{array}{l}\text { Starters mean age } 40.40 \text { years; } \\
\text { completers mean age } 47.68 \\
\text { years; adults with prehyperten- } \\
\text { sion or hypertension }\end{array}$ & 24 weeks & $\begin{array}{l}\text { Weight change, BMI change, DBP } \\
\text { change, SBP change, hypertension } \\
\text { category change }\end{array}$ & No \\
\hline
\end{tabular}




\begin{tabular}{|c|c|c|c|c|c|c|}
\hline Study & Country & Sample size & $\begin{array}{l}\text { Participants' demographic and } \\
\text { hypertension characteristics }\end{array}$ & Duration & Outcomes & Theory \\
\hline $\begin{array}{l}\text { Weerahandi et } \\
\text { al [52] }\end{array}$ & $\begin{array}{l}\text { United } \\
\text { States }\end{array}$ & 17 & $\begin{array}{l}\text { Mean age } 59 \text { years; adults cur- } \\
\text { rently taking hypertension } \\
\text { medication and had a diagnosis } \\
\text { of prehypertension or stage } 1 \\
\text { hypertension }\end{array}$ & $\begin{array}{l}13 \text { weeks or } 120 \\
\text { days }\end{array}$ & $\begin{array}{l}\text { Engagement and acceptability: the } \\
\text { number of blood pressure measure- } \\
\text { ments, weight measurements, and } \\
\text { daily steps were logged; the number } \\
\text { of coaching phone calls attempted and } \\
\text { completed, servings documented in } \\
\text { the dietary assessment, and goals set } \\
\text { were also assessed. Physiological pa- } \\
\text { rameters: BP, heart rate, weight, and } \\
\text { steps changes }\end{array}$ & No \\
\hline
\end{tabular}

${ }^{\mathrm{a}} \mathrm{SBP}$ : systolic blood pressure.

${ }^{\mathrm{D}} \mathrm{DBP}$ : diastolic blood pressure.

${ }^{\mathrm{c}} \mathrm{BP}$ : blood pressure.

${ }^{\mathrm{d}} \mathrm{IG}$ : intervention group.

e PO: primary outcome.

${ }^{\mathrm{f}} \mathrm{SO}$ : secondary outcome.

$\mathrm{g}_{\mathrm{MA}}$ : medication adherence.

${ }^{\mathrm{h}} \mathrm{CG}$ : control group.

\section{Intervention Strategies}

\section{Overview}

All of the studies used at least two strategies to engage patients.

The number of strategies used in the interventions varied from

2 to 6 , with a possible maximum of 8 . Table 2 provides further details. 
Table 2. Engagement strategies used in the interventions.

\begin{tabular}{|c|c|c|c|c|c|c|c|c|}
\hline Study & $\begin{array}{l}\text { Providing } \\
\text { health-related } \\
\text { educational in- } \\
\text { formation }\end{array}$ & $\begin{array}{l}\text { Reminding } \\
\text { or alerting } \\
\text { users }\end{array}$ & $\begin{array}{l}\text { Motivational } \\
\text { messages or } \\
\text { encourage- } \\
\text { ment }\end{array}$ & $\begin{array}{l}\text { Recording } \\
\text { and tracking } \\
\text { health infor- } \\
\text { mation }\end{array}$ & $\begin{array}{l}\text { Providing guid- } \\
\text { ance based on in- } \\
\text { formation entered } \\
\text { by the user }\end{array}$ & $\begin{array}{l}\text { Enabling 2-way } \\
\text { communication } \\
\text { with clinicians }\end{array}$ & $\begin{array}{l}\text { Providing sup- } \\
\text { port through } \\
\text { social net- } \\
\text { works }\end{array}$ & $\begin{array}{l}\text { Supporting be- } \\
\text { havior change } \\
\text { through re- } \\
\text { wards }\end{array}$ \\
\hline $\begin{array}{l}\text { Bengtsson et } \\
\text { al [31] }\end{array}$ & No & Yes & Yes & Yes & No & No & No & No \\
\hline $\begin{array}{l}\text { Chandler et al } \\
\text { [38] }\end{array}$ & No & Yes & Yes & Yes & Yes & No & No & No \\
\hline $\begin{array}{l}\text { Ciemins et al } \\
{[49]}\end{array}$ & Yes & Unknown & No & Yes & Yes & No & No & No \\
\hline $\begin{array}{l}\text { Davidson et al } \\
\text { [39] }\end{array}$ & No & Yes & Yes & No & No & No & No & No \\
\hline $\begin{array}{l}\text { Duan et al } \\
\text { [43] }\end{array}$ & Yes & Yes & No & Yes & Yes & No & $\begin{array}{l}\text { Yes (leader- } \\
\text { board module, } \\
\text { version 4) }\end{array}$ & No \\
\hline $\begin{array}{l}\text { Gong et al } \\
{[44]}\end{array}$ & No & Yes & No & Yes & Yes & Yes & No & No \\
\hline $\begin{array}{l}\text { Hallberg et al } \\
\text { [32] }\end{array}$ & No & Yes & Yes & Yes & No & No & No & No \\
\hline $\begin{array}{l}\text { Kang and Park } \\
{[25]}\end{array}$ & Yes & Yes & No & Yes & Yes & No & No & No \\
\hline $\begin{array}{l}\text { Kaplan et al } \\
\text { [48] }\end{array}$ & Yes & Yes & Yes & Yes & Yes & No & No & Yes \\
\hline Mao et al [26] & Yes & Yes & Yes & Yes & Yes & Yes & No & No \\
\hline $\begin{array}{l}\text { Márquez Con- } \\
\text { treras [45] }\end{array}$ & No & Yes & No & Yes & No & No & No & No \\
\hline $\begin{array}{l}\text { McGillicuddy } \\
\text { et al [28] }\end{array}$ & No & Yes & No & Yes & Yes & No & No & No \\
\hline $\begin{array}{l}\text { Moore et al } \\
{[37]}\end{array}$ & No & No & No & Yes & No & Yes & No & No \\
\hline $\begin{array}{l}\text { Morawski et } \\
\text { al [27] }\end{array}$ & No & Yes & No & Yes & No & No & Yes & No \\
\hline $\begin{array}{l}\text { Ovbiagele et } \\
\text { al [47] }\end{array}$ & No & Yes & Yes & Yes & Yes & No & No & No \\
\hline Patel et al [51] & Yes & Yes & No & Yes & No & No & No & No \\
\hline $\begin{array}{l}\text { Persell et al } \\
{[36]}\end{array}$ & Yes & Yes & Yes & Yes & Yes & $\begin{array}{l}\text { Artificial intelli- } \\
\text { gence coaching }\end{array}$ & No & No \\
\hline $\begin{array}{l}\text { Petrella et al } \\
\text { [46] }\end{array}$ & No & No & No & Yes & Yes & No & No & No \\
\hline $\begin{array}{l}\text { Thies et al } \\
\text { [14] }\end{array}$ & No & No & No & Yes & Yes & Yes & No & No \\
\hline $\begin{array}{l}\text { Toro-Ramos } \\
\text { et al [50] }\end{array}$ & Yes & Yes & Yes & Yes & Yes & Yes & No & No \\
\hline $\begin{array}{l}\text { Weerahandi et } \\
\text { al [52] }\end{array}$ & Yes & No & No & Yes & Yes & Yes & No & No \\
\hline
\end{tabular}

\section{Providing Health-Related Educational Information}

A total of 9 studies $[25,26,36,43,48-52]$ provided health-related educational information. The content of education varied, with some studies [36,43,48,49] focusing on hypertension, some studies [25,51] focusing on hypertensive medications, and some studies (eg, the studies by Mao et al [26], Toro-Ramos et al [50], and Weerahandi et al [52]) including dietary approaches to reducing hypertension. However, of the 9 studies, only 1 (11\%) [36] specified in the educational materials the reason why self-monitoring is important in BP management and how their control through healthy behavior change is important for lowering the risk of complications. 


\section{Recording and Tracking Health Information}

Some studies [14,25,27,28,31,32,36-38,43,44,47] included features to record both BP and medication adherence or intake. Some other studies included features to record either BP [45,46,48-50,52] or medication intake or adherence [51]. Some studies also recorded other information such as medication side effects (eg, the studies by Bengtsson et al [31] and Hallberg et al [32]), symptom logging (eg, the studies by Bengtsson et al [31], Hallberg et al [32], and Duan et al [43]), and the tracking of diet, heart rate, weight, and steps (eg, the study by Weerahandi et al [52]).

\section{Reminding or Alerting Users}

Most studies [25,27,28,36,38,39,43-45,47,48,50,51] included reminders for medication intake or BP monitoring, or both. Various reminders focusing on other topics or other types were exercise [44]; weight, diet, exercise, and discomfort [43]; weight, meals and snacks, and physical activity [36]; hospital visit date and input of lifestyle data [25]; clients' personal goals [26]; appointments [45]; or alerting a Medfriend who provides peer support when doses are missed [27]. However, some studies $[31,32]$ included reminders but did not specify the content of reminders.

\section{Motivational Messages or Encouragement}

There are studies that included motivational messages or encouragement $[26,31,32,36,38,39,47,48,50]$. For instance, the motivational messages of 1 intervention [38] were designed based on participants' previous medication adherence levels (ie, nonadherence, partial adherence, and complete adherence) and on their values, beliefs, and long-term or short-term life goals.

\section{Providing Support Through Social Networks}

A total of 2 studies included the feature to provide support through social networks. In version 4 of 1 app [43], a leaderboard module presenting and comparing the scores generated based on each patients' self-management behaviors was provided for those users who wanted to enhance their self-management motivation. In another study [27], users were able to designate a Medfriend, who was someone else who was granted access to the patient's medication taking history, received alerts when the patient missed doses, and was able to provide peer support.

\section{Supporting Behavior Change Through Rewards}

Only 1 intervention [48] included gamification features with a reward system to maximize user interaction. In this app, enthusiastic amination appeared on the screen after each BP recording event [48].

\section{Providing Guidance Based on Information Entered by the Users}

If cutoffs of BP were exceeded or out-of-range values were observed, patients were contacted [47] or were recommended to take additional BP measurements $[28,36,44]$ or to seek medical attention $[44,50]$. Health care providers were notified [49] or were called when extreme values were recorded [36] or contacted to follow-up with participants $[43,46]$ and asked to determine the course of action to take with the participant [38] or to make an adjustment to medical regimen as warranted $[28,47]$. In addition to guidance on out-of-range values, the interventions also helped with solving problems [52]; providing personalized or tailored recommendations or advice [26,36,44]; providing encouragement and suggestions and answering nonpressing questions [14]; providing personalized explanations regarding the stages of hypertension and translation into cardiovascular risk [48]; providing strategies to address behavior change related to calorie reduction, diet improvement, nutrient intake, physical activity increase, and sodium intake reduction [50]; or providing tailored recommendations to users' questions on lifestyle management (ie, sodium intake, body weight, waist circumference, exercise, alcohol, smoking, and stress) [25].

\section{Interactivity}

Interactivity was analyzed based on providing guidance related to information entered by the users (eg, when abnormal BP values were recorded) and based on whether or not the intervention enabled 2-way regular communication (outside of just specific situations such as when abnormal BP values are observed) between users and a health care professional. A total of 9 studies [28,36,38,43,44,46,47,49,50] included 1-way communication under possible critical conditions. Patients or their health care providers were notified or contacted when out-of-range BP values were reported. Interactivity was limited in the interventions; only $33 \%$ (7/21) of the studies provided the possibility of interaction with health care providers or health coaches. In terms of 2-way communication, studies included the possibility of communicating with physicians [14,44], health coaches [26,37,50,52], or an artificial intelligence coach [36]. Users were able to have remote consultations with professional doctors [44] or members of their care team [14]. For instance, a trained coach [50], professionals (licensed nutritionists, physical therapists, and social workers) [26], or master clinicians [37] provided human coaching. For a summary of interactivity, please see Table 3. 
Table 3. Interactivity, tailoring, and engagement.

\begin{tabular}{|c|c|c|c|c|}
\hline Study & Interactivity & Tailoring & Microlevel engagement & Macrolevel engagement \\
\hline Bengtsson et al [31] & No & Yes & No & No \\
\hline Chandler et al [38] & Yes & Yes & No & No \\
\hline Ciemins et al [49] & Yes & No & No & No \\
\hline Davidson et al [39] & No & Yes & No & No \\
\hline Duan et al [43] & Yes & Yes & No & No \\
\hline Gong et al [44] & Yes & Yes & No & No \\
\hline Hallberg et al [32] & No & Yes & No & No \\
\hline Kang and Park [25] & No & Yes & No & No \\
\hline Kaplan et al [48] & No & Yes & Yes & No \\
\hline Mao et al [26] & Yes & Yes & Yes & No \\
\hline Márquez Contreras [45] & No & No & No & No \\
\hline McGillicuddy et al [28] & Yes & Yes & No & No \\
\hline Moore et al [37] & Yes & Yes & No & No \\
\hline Morawski et al [27] & No & Yes & No & No \\
\hline Ovbiagele et al [47] & Yes & Yes & No & No \\
\hline Patel et al [51] & No & Yes & No & No \\
\hline Persell et al [36] & Yes & Yes & No & No \\
\hline Petrella et al [46] & Yes & Yes & No & No \\
\hline Thies et al [14] & Yes & No & No & No \\
\hline Toro-Ramos et al [50] & Yes & Yes & Yes & No \\
\hline Weerahandi et al [52] & Yes & Yes & Yes & No \\
\hline
\end{tabular}

\section{Tailoring, Customization, or Personalization}

Some level of tailoring was achieved in many studies. All 21 studies included only 1 app version, except 1 (5\%) study [43]. Moreover, 4 versions of the app were developed based on users' disease cognition, self-management experience, and self-management motivation, wherein version 1 had three functional modules (ie, management plan, reminder service, and health checkup), version 2 had four modules (health education was added), version 3 had five modules (health education and health report were added), and version 4 had all six modules (health education, health report, and health report were added) [43].

Some studies [26,46,52] included personalized health goals, such as individualized exercise prescription [46]. The content, information, or features of some interventions was or were customized, based on goals or individual preferences [27,43,50,51], based on antihypertensive medication prescription [31], and based on users' values and inputs [36].

Some studies $[43,44,48]$ provided personalized feedback or advice in the intervention. For instance, in 1 study, physician's advice was based on patient's hypertension self-management experience level [43], and in another study, a personalized explanation of the relationship between stages of hypertension and cardiovascular risk was provided [48]. Interventions with tailored target management recommendations included the studies by Kang and Park [25] and Persell et al [36]. In the study by Moore et al [37], users could make shared decisions about diet, exercise, stress management, and medication with the coach. In some studies, the motivational messages were tailored based on users' personal preferences [31] and on users' medication adherence rates, goals, and values and beliefs $[38,39]$. A total of 2 studies [28,32] included tailored reminders. In some studies $[28,47,52]$, the communication model or channel was customized so that patients were contacted via the preferred mode: SMS text messaging, email, or phone. In 1 study [26], the intervention dose (eg, coaching frequencies) was based on the participants' needs and availability. For a summary of tailoring, please see Table 3.

\section{Engagement}

In addition to describing the intervention strategies used to engage users, we also explored how engagement has been defined, reflected, and related to biomedical outcomes.

\section{How Engagement Has Been Defined}

Engagement was defined or operationalized as microlevel interactions with the interventions, but from different dimensions, such as frequency of engagement, time or duration of engagement with the app, and intensity of engagement. A total of 4 studies $[26,48,50,52]$ clearly defined or operationalized engagement (Table 3). Moreover, 1 study [48] defined low engagement (ie, "recording BP for less than 4 weeks"), medium engagement (ie, "recording BP for 4-8 weeks"), and high engagement (ie, "recording BP for longer than 8 weeks"). 
Another study [26] also defined low (ie, "at the bottom quartile of number of messages and video consults"), medium (ie, "participants in the 25th-75th engagement percentiles"), and high engagement (ie, "top quartile of messages sent per month or number of coaching consults in the 4-month coaching period"). Toro-Ramos et al [50] defined different levels of engagement as starters (ie, "participants who completed at least one lesson per week during the first month, as well as engaged with the health coach (at least once through in app one-on-one messages or through phone calls)"), and completers (ie, "participants who completed at least nine core lessons of 22"). Weerahandi et al [52] defined engagement as "Messages sent to the coach per person, messages sent from the coach per person," "number of times blood pressure was logged," "number of times weight was logged," "number of times steps were logged," "logged food entries," and "goals recorded."

None of the studies we reviewed directly examined or measured users' subjective experience of engagement, focusing on attention, interest, and affect. Some studies explored users' subjective experience with a focus on user satisfaction or usability in general using interviews or surveys $[38,49]$, usually conducted at the end of the intervention, which may not objectively capture attention or affect during the intervention, given the broad focus and retrospective nature [53].

\section{Behaviors Reflecting Engagement}

Although some studies did not clearly state in the articles that they measured engagement with digital behavior change interventions (microlevel) or engagement with behavior change (macrolevel), those behavior-related outcome variables, to some extent, reflected users' macro- or microlevel of engagement, or both. Two commonly measured behaviors in the outcome variables of the studies were medication adherence and BP self-monitoring. Studies measuring medication adherence used different methods: using technology or devices [38,39,45,51], using self-report or surveys [25,27,36,38,44,51], or using a pharmacy refill rate [51]. Studies [36,38,39,43,46,48,49,52] also used the app or a Bluetooth BP device to measure BP self-monitoring behavior.

In addition to medication adherence and BP self-monitoring, other behaviors were also measured: food or meals logged $[36,50,52]$, messages sent or conversations with the app $[14,26,36,52]$, steps taken $[46,52]$, body weight logging [46,52], frequency of users accessing their weekly BP report [48], number of coaching consults [26], and lessons completed [50].

\section{The Relationship Between Engagement and Biomedical Outcomes}

Of the 4 studies that clearly defined engagement, 3 (75\%) studies $[26,48,50]$ tested and demonstrated the statistical relationship between engagement and biomedical outcomes (ie, weight or BP change), indicating that higher engagement was associated with significantly better biomedical outcomes. However, in 1 study [52], the relationship between levels of engagement and biomedical outcomes was not tested. Among studies that did not explicitly define engagement but included behaviors reflecting engagement, none statistically tested the relationship between the behaviors and biomedical outcomes. However, using patient interviews, 1 study [32] explained the mechanism between engagement and the motivation for macrolevel behavior change: as patients became engaged in graphs or through answering questions and measuring their BP, they were motivated to follow their treatment and understood the interplay between lifestyle and BP. Persell et al [36] did not test engagement but tested the factor crucial to macrolevel engagement or behavior change, self-efficacy [54], or engagement self-efficacy [55], that is, the self-confidence in using the app, controlling BP, knowing when medication changes were needed, and performing nonpharmacologic behaviors to control BP. The study by Persell et al [36] found that self-efficacy in controlling BP was greater in the intervention group.

\section{Discussion}

\section{Principal Findings}

\section{Overview}

To the authors' knowledge, this is the first review examining the interactivity, customization, and engagement factors of mHealth interventions for hypertension self-management. This review included 21 studies.

\section{Participants}

On the basis of the results of the participant inclusion criteria, some studies had very specific criteria, whereas others had very broad inclusion criteria. As participant characteristics (eg, disease type and severity of disease) were quite diverse in some studies, more research is needed to explore the goals, needs, and characteristics of users.

\section{Design of Interventions}

Engagement or self-management behaviors were not included in the outcomes of the limited longitudinal studies. No studies tested the engagement or self-management behaviors after the mHealth technology was no longer provided. Lack of longitudinal design leads to inability to elucidate behavior change or engagement patterns over time. Without testing macrolevel engagement or self-management behaviors (eg, medication adherence) when interventions or apps are no longer available, it cannot be confirmed that digital behavior change interventions are effective in changing behaviors in the long run.

\section{Theoretical Frameworks Applied}

Self-determination theory, health belief model, technology acceptance model, and technology-supported apprenticeship models have been applied to a limited number of studies. Some of these theories (eg, self-determination theory) have also been applied to diabetes self-management interventions using mobile apps [56] and applied to mHealth interventions in improving medication adherence among people with hypertension [11]. More interventions should adopt a theoretical framework (eg, behavior change theories) to guide work in this area.

\section{Optimal Combination of Engagement Strategies}

Many studies have used a combination of features that are likely to engage users. What specific combination of features works 
best for engagement is yet to be determined, but should consider patients' characteristics. For instance, patient motivation for self-management may be a factor to consider. Providing patient education content in an app can be a way of engaging patients, especially those who are motivated. However, for those who are not motivated (eg, "motivating participants to read the educational materials remained a challenge," as indicated by Weerahandi et al [52]), a patient education section may be less beneficial, and other strategies should be considered to motivate patients. Similarly, reminders and motivational messages could be more effective when refined according to patient characteristics and interactions with the app [32,51].

\section{Interactivity}

Interactivity was limited in digital behavior change interventions, as only 7 interventions provided 2-way communication between users and a health care professional or a coach. Moreover, 9 interventions provided 1-way communication in possible critical conditions; that is, when abnormal BP values were recorded, users or health care professionals were notified. On the basis of the results, the levels of interactivity between users and health care professionals can be characterized into four major categories: no interaction, limited interaction, regular interaction, or focused interaction. Limited interaction includes providing support under possible critical conditions. Regular interaction includes providing the possibility of 2-way communication between users and health care providers (eg, questions and answers and receiving regular feedback and recommendations), along with other strategies or app features. Focused interaction includes providing patient coaching by a clinician or a trained coach, which is the dominant feature, goal of the app, or intervention.

In our review, we found that some apps contained interactivity functions, whereas others did not. Authors did not describe the decision to exclude interactive features; we posit that the decision of including or excluding interactivity might be based on a variety of factors, including patient factors (eg, needs), intervention goals, and health care providers' availability. For instance, if an app's aim is medication adherence, interactivity is not a very important feature, whereas if an app's aim is logging symptoms, especially alarming symptoms, then interactivity (eg, health care providers' feedback) would be a critical function. In addition, provider-related factors are also worth considering. Studies have demonstrated health care providers' barriers of using apps to communicate with patients, including time constraints, increased workload, lack of interest, and lack of investment in app development [57].

Some level of potential interactivity should be included in the interventions using mobile apps for hypertension self-management. As 1 article examining patient perspectives indicated, ambiguity and anxiety could be provoked with BP readings, especially when readings are high [7]. Interaction with health care professionals, at least during possible critical points perceived by patients, can be an essential feature to provide professional guidance and ease the concerns and promote engagement with the interventions and the behavior change process [58].

\section{Tailoring}

The tailoring of interventions varied at different aspects, from tailoring of intervention content (including goals, patient education, advice and feedback from health professionals, reminders, and motivational messages) to tailoring intervention dose and communication mode. Tailoring was carried out in several ways, including consideration of patient characteristics such as goals, preferences, disease characteristics (eg, hypertension stage and medication list), disease self-management experience level, medication adherence rate, values, and beliefs. Although multiple studies included reminders for medication administration, only two of them provided tailored reminders. Medication nonadherence can be due to many factors: medication side effects, cost, forgetfulness, or perceived lack of need to take medications. In addition, personal and cultural values and beliefs (eg, perception of illness, illness knowledge, health literacy, cultural beliefs, self-efficacy, and spiritual and religious beliefs [59]) can impact medication adherence. For instance, higher perceived benefits of herbs and lower perceived benefits of Western medications were predictors of antihypertensive medication nonadherence among Chinese immigrants [60]. A medication reminder feature may therefore work well for those who forget to take medications but may be ineffective for those who do not take medications as prescribed because of costs or potential side effects [61] or those who do not believe in the benefits of Western medications. For those who have high medication adherence rates, reminder features may be redundant or perceived as annoying. To promote medication adherence, intervention content and approach should be tailored according to such personal characteristics. Providing an app with various modules or features and giving the users the ability to select among them may represent an optimal solution for potentially diverse population.

\section{Engagement}

Engagement was defined or operationalized as a microlevel interaction with the interventions but from different dimensions, such as frequency of engagement, time or duration of engagement with the program, and intensity of engagement. There are a couple of possible reasons why subjective experience of engagement such as attention, affect, and interest were not examined in the studies. Self-report of engagement (subjective measure) at multiple points during the intervention or app use may be disrupting [53] and may create excessive burden for users, especially when they are asked to periodically perform different tasks (eg, logging symptoms and BP and reporting medication adherence) using the app. In addition, objective measures (eg, app use data) can be used to capture attention, interest, or affect during the app use or intervention [55]. For instance, mouse cursor tracking or eye tracking can be used to measure attention [62]. Similarly, digital health intervention use (eg, time spent on education content page and messages sent to the health coach) can be used to measure attention or interest in a nonobstructive way or, to some extent, reflect attention or interest. These could be the reasons why studies used data use to measure engagement but seldom used subjective measure to directly measure attention, affect, or interest. 
For all studies that tested this relationship, higher engagement was associated with better biomedical outcomes. However, this result should be interpreted with caution because, first, the result is from 3 applicable studies and, second, although the studies are valuable to the engagement literature, they might have some risk of bias.

Although most studies did not define or focus on engagement per se, their outcome variables reflected engagement with digital behavior change interventions. When reviewing studies of engagement in mHealth, authors should consider seeking out both studies that explicitly mention engagement, as well as studies that do not explicitly define engagement but examine behaviors that reflect engagement.

No studies tested macrolevel engagement directly, but 1 study [36] tested a factor crucial to macrolevel and microlevel engagement or behavior change: self-efficacy or self-confidence (eg, using the app, performing nonpharmacologic behaviors to control BP). In 1 study [7], researchers found that patients with hypertension have various levels of digital competence (defined as "becoming familiar and comfortable with using technology to manage hypertension") in that some were not interested in using apps for hypertension management and others were digitally competent to use apps. In another study focusing on African American older adults, "participants expressed concerns about not being informed or trained sufficiently to integrate technology for hypertension self-management" [63]. These findings imply that self-efficacy, especially self-efficacy in using technology for disease self-management, can be another important patient factor to be considered when designing interventions $[54,55,64]$.

\section{Our Patient-Centered Framework}

Overall, the results of this study agreed with those of a prior systematic review [65] of mHealth for self-management of cardiometabolic risk factors, in that some studies are theoretically driven, while direct measurement and evaluation of engagement was limited.

Considering the definition of engagement [4], the motivational technology model [20], and the results of the review, a framework for the use of mHealth technology for hypertension self-management is proposed (Figure 2). This patient-centered engagement framework emphasizes the important role of patient-centered factors, including but not limited to disease factors, self-management factors, users' personal preferences, cultural factors, and behaviors related to disease self-management. These factors determine the aspects of an intervention to be tailored and determine the level of interactivity. Patient-centered factors, together with tailoring and interactivity level, determine engagement and subsequently intervention efficacy in improving biomedical outcomes. For instance, Thies et al [14] concluded that their failed intervention was due to lack of attention to patients' eHealth literacy (defined as "the ability to seek, find, understand, and appraise health information from electronic sources and apply the knowledge gained to addressing or solving a health problem") [66] and lack of proficiency regarding the chronic disease type. Whereas other engagement frameworks [15] focus on strategies (eg, providing medical information, sending reminders, and tracking health data) to engage patients, our framework highlights categories of patient factors to be considered and how those factors are crucial to customization, interactivity, and engagement.

Figure 2. Patient-centered engagement framework for hypertension self-management using mobile health (mHealth) technology.

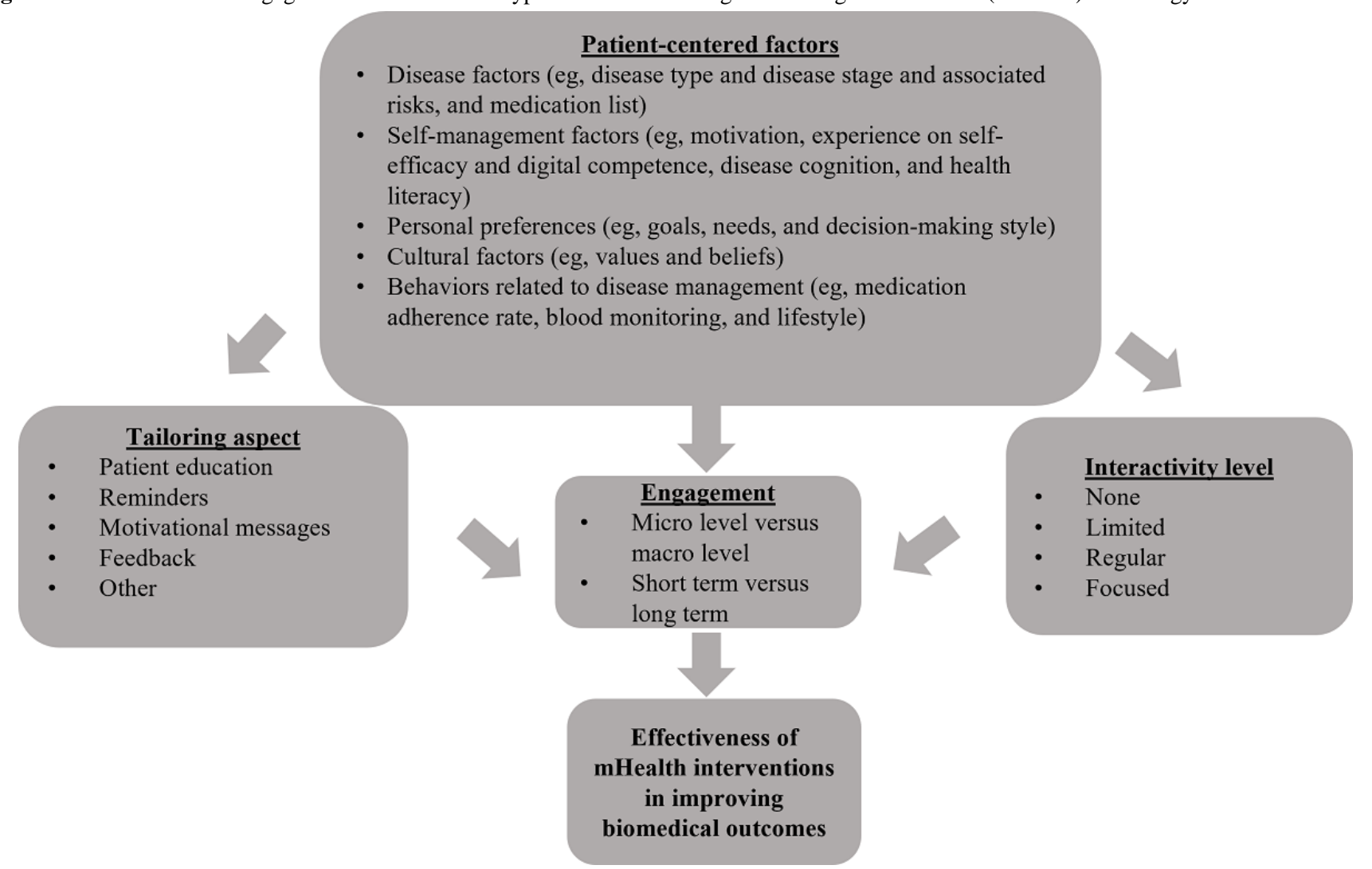




\section{Limitations}

There are some limitations of this review. Only studies published in English were included in the review, and therefore, there remains potential neglect of important studies published in other languages. Although the authors used a systematic search strategy, other studies meeting the inclusion criteria may have been missed. For instance, those studies not including the key search terms used in the systematic search might be excluded. We were unable to conduct a meta-analysis, owing to the heterogeneity of the studies and outcomes. Further, other factors (eg, navigability) that are also important for engagement and efficacy of interventions were not examined in this review. Given that the proposed patient-centered framework was based on the results of the studies included in the review, it is possible that there are other patient-centered factors (eg, outcome expectation, a significant factor of engagement [55]) that could be important for engagement in the context of hypertension self-management that are not included in the framework.

\section{Conclusions}

Among mHealth app interventions focused on hypertensive management, engagement, interactivity, and tailoring have been implemented in various ways, as demonstrated by the 21 studies included in this review. The authors examined several strategies used to facilitate engagement. The results support the essential roles of engagement in intervention effectiveness and the essential roles of patient factors in tailoring, interactivity, and engagement. A patient-centered engagement framework for hypertension self-management using mHealth technology was proposed, with the intent to help facilitate intervention design and disease self-management using mHealth technology in the future.

\section{Acknowledgments}

This work was supported by National Cancer Institute of National Institutes of Health (NIH; grant U01CA248240 to LL), by Eunice Kennedy Shriver National Institute of Child Health and Human Development of NIH (grant P30HD106451 to LL), by National Heart, Lung, and Blood Institute of NIH (grant K23HL155890 to DA), and by an American Heart Association-Robert Wood Johnson Foundation Faculty Development Program Grant (to DA).

\section{Authors' Contributions}

WC, MWM, and LL conceived the study. WC performed the systematic search. WC and XL screened and coded the studies included for the review. WC analyzed the data and drafted the manuscript. All authors interpreted the results and revised the manuscript critically. All authors gave their final approval of the manuscript.

\section{Conflicts of Interest}

None declared.

\section{Multimedia Appendix 1}

Search strategy.

[DOCX File, 14 KB-Multimedia Appendix 1]

\section{Multimedia Appendix 2}

Risk of bias assessment for randomized control trails.

[DOCX File, 15 KB-Multimedia Appendix 2]

\section{Multimedia Appendix 3}

Risk of bias assessment for observational studies.

[DOCX File, 15 KB-Multimedia Appendix 3]

\section{Multimedia Appendix 4}

Risk of bias assessment for pre-post studies without a control group.

[DOCX File, $15 \mathrm{~KB}$-Multimedia Appendix 4]

\section{Multimedia Appendix 5}

Risk of bias assessment for qualitative studies.

[DOCX File, 14 KB-Multimedia Appendix 5]

\section{References}

1. Facts about hypertension. Centers for Disease Control and Prevention. 2021. URL: https://www.cdc.gov/bloodpressure/ facts.htm [accessed 2021-03-15] 
2. Tuberculosis: global task force on digital health: frequently asked questions. World Health Organization. 2015. URL: https:/ /www.who.int/news-room/q-a-detail/tuberculosis-global-task-force-on-digital-health [accessed 2020-12-30]

3. Perski O, Blandford A, West R, Michie S. Conceptualising engagement with digital behaviour change interventions: a systematic review using principles from critical interpretive synthesis. Transl Behav Med 2017;7(2):254-267 [FREE Full text] [doi: 10.1007/s13142-016-0453-1] [Medline: 27966189]

4. Yardley L, Spring BJ, Riper H, Morrison LG, Crane DH, Curtis K, et al. Understanding and promoting effective engagement with digital behavior change interventions. Am J Prev Med 2016;51(5):833-842. [doi: 10.1016/j.amepre.2016.06.015] [Medline: 27745683]

5. Andre N, Wibawanti R, Siswanto BB. Mobile phone-based intervention in hypertension management. Int J Hypertens 2019;2019:9021017 [FREE Full text] [doi: 10.1155/2019/9021017] [Medline: 31080670 ]

6. Liu K, Xie Z, Or CK. Effectiveness of mobile app-assisted self-care interventions for improving patient outcomes in type 2 diabetes and/or hypertension: systematic review and meta-analysis of randomized controlled trials. JMIR Mhealth Uhealth 2020;8(8):e15779 [FREE Full text] [doi: 10.2196/15779] [Medline: 32459654]

7. Morrissey EC, Casey M, Glynn LG, Walsh JC, Molloy GJ. Smartphone apps for improving medication adherence in hypertension: patients' perspectives. Patient Prefer Adherence 2018;12:813-822 [FREE Full text] [doi: 10.2147/PPA.S145647] [Medline: 29785096]

8. Alessa T, Hawley MS, Hock ES, de Witte L. Smartphone apps to support self-management of hypertension: review and content analysis. JMIR Mhealth Uhealth 2019;7(5):e13645 [FREE Full text] [doi: 10.2196/13645] [Medline: $\underline{31140434]}$

9. Alessa T, Abdi S, Hawley MS, de Witte L. Mobile apps to support the self-management of hypertension: systematic review of effectiveness, usability, and user satisfaction. JMIR Mhealth Uhealth 2018;6(7):e10723 [FREE Full text] [doi: 10.2196/10723] [Medline: $\underline{30037787]}$

10. Li R, Liang N, Bu F, Hesketh T. The effectiveness of self-management of hypertension in adults using mobile health: systematic review and meta-analysis. JMIR Mhealth Uhealth 2020;8(3):e17776 [FREE Full text] [doi: 10.2196/17776] [Medline: $\underline{\text { 32217503] }}$

11. Xiong S, Berkhouse H, Schooler M, Pu W, Sun A, Gong E, et al. Effectiveness of mHealth interventions in improving medication adherence among people with hypertension: a systematic review. Curr Hypertens Rep 2018;20(10):86. [doi: 10.1007/s11906-018-0886-7] [Medline: 30088110]

12. Donkin L, Christensen H, Naismith SL, Neal B, Hickie IB, Glozier N. A systematic review of the impact of adherence on the effectiveness of e-therapies. J Med Internet Res 2011;13(3):e52 [FREE Full text] [doi: 10.2196/jmir.1772] [Medline: 21821503]

13. Serrano KJ, Coa KI, Yu M, Wolff-Hughes DL, Atienza AA. Characterizing user engagement with health app data: a data mining approach. Transl Behav Med 2017;7(2):277-285 [FREE Full text] [doi: 10.1007/s13142-017-0508-y] [Medline: 28616846]

14. Thies K, Anderson D, Cramer B. Lack of adoption of a mobile app to support patient self-management of diabetes and hypertension in a federally qualified health center: interview analysis of staff and patients in a failed randomized trial. JMIR Hum Factors 2017;4(4):e24 [FREE Full text] [doi: 10.2196/humanfactors.7709] [Medline: 28974481]

15. Shapiro-Mathews E, Barton AJ. Using the patient engagement framework to develop an institutional mobile health strategy. Clin Nurse Spec 2013;27(5):221-223. [doi: 10.1097/NUR.0b013e3182a0b9e2] [Medline: 23942098]

16. Singh K, Drouin K, Newmark LP, Rozenblum R, Lee J, Landman A, et al. Developing a framework for evaluating the patient engagement, quality, and safety of mobile health applications. Issue Brief (Commonw Fund) 2016;5:1-11. [Medline: 26934758]

17. Nelson LA, Coston TD, Cherrington AL, Osborn CY. Patterns of user engagement with mobile- and web-delivered self-care interventions for adults with T2DM: a review of the literature. Curr Diab Rep 2016;16(7):66 [FREE Full text] [doi: 10.1007/s11892-016-0755-1] [Medline: 27255269]

18. Pham Q, Graham G, Carrion C, Morita PP, Seto E, Stinson JN, et al. A library of analytic indicators to evaluate effective engagement with consumer mHealth apps for chronic conditions: scoping review. JMIR Mhealth Uhealth 2019;7(1):e11941 [FREE Full text] [doi: 10.2196/11941] [Medline: 30664463]

19. Short CE, DeSmet A, Woods C, Williams SL, Maher C, Middelweerd A, et al. Measuring engagement in eHealth and mHealth behavior change interventions: viewpoint of methodologies. J Med Internet Res 2018;20(11):e292 [FREE Full text] [doi: 10.2196/jmir.9397] [Medline: 30446482]

20. Sundar SS, Bellur SS, Jia H. Motivational technologies: a theoretical framework for designing preventive health applications. In: Proceedings of 7th International Conference on Persuasive Technology. 2012 Presented at: PERSUASIVE '12; June 6-8, 2012; Linköping, Sweden p. 112-122. [doi: 10.1007/978-3-642-31037-9 10]

21. Sundar SS. The handbook of the psychology of communication technology. Hoboken, NJ: Wiley; 2015.

22. Sahin C, Courtney KL, Naylor PJ, E Rhodes R. Tailored mobile text messaging interventions targeting type 2 diabetes self-management: a systematic review and a meta-analysis. Digit Health 2019;5:2055207619845279 [FREE Full text] [doi: 10.1177/2055207619845279] [Medline: 31041110]

23. König LM, Attig C, Franke T, Renner B. Barriers to and facilitators for using nutrition apps: systematic review and conceptual framework. JMIR Mhealth Uhealth 2021;9(6):e20037 [FREE Full text] [doi: 10.2196/20037] [Medline: $\underline{34254938]}$ 
24. Al-Noumani H, Wu JR, Barksdale D, Sherwood G, AlKhasawneh E, Knafl G. Health beliefs and medication adherence in patients with hypertension: a systematic review of quantitative studies. Patient Educ Couns 2019;102(6):1045-1056. [doi: 10.1016/j.pec.2019.02.022] [Medline: 30846205]

25. Kang H, Park HA. A mobile app for hypertension management based on clinical practice guidelines: development and deployment. JMIR Mhealth Uhealth 2016;4(1):e12 [FREE Full text] [doi: 10.2196/mhealth.4966] [Medline: 26839283]

26. Mao AY, Chen C, Magana C, Caballero Barajas K, Olayiwola JN. A mobile phone-based health coaching intervention for weight loss and blood pressure reduction in a national payer population: a retrospective study. JMIR Mhealth Uhealth 2017;5(6):e80 [FREE Full text] [doi: 10.2196/mhealth.7591] [Medline: 28596147]

27. Morawski K, Ghazinouri R, Krumme A, Lauffenburger JC, Lu Z, Durfee E, et al. Association of a smartphone application with medication adherence and blood pressure control: the MedISAFE-BP randomized clinical trial. JAMA Intern Med 2018;178(6):802-809 [FREE Full text] [doi: 10.1001/jamainternmed.2018.0447] [Medline: 29710289]

28. McGillicuddy JW, Taber DJ, Mueller M, Patel S, Baliga PK, Chavin KD, et al. Sustainability of improvements in medication adherence through a mobile health intervention. Prog Transplant 2015;25(3):217-223 [FREE Full text] [doi: 10.7182/pit2015975] [Medline: 26308780]

29. Covidence systematic review software. Melbourne, Australia: Veritas Health Innovation URL: https://www.covidence.org/ [accessed 2021-07-30]

30. Bengtsson U, Kjellgren K, Höfer S, Taft C, Ring L. Developing an interactive mobile phone self-report system for self-management of hypertension. Part 2: content validity and usability. Blood Press 2014;23(5):296-306 [FREE Full text] [doi: 10.3109/08037051.2014.901009] [Medline: 24786778]

31. Bengtsson U, Kjellgren K, Hallberg I, Lindwall M, Taft C. Improved blood pressure control using an interactive mobile phone support system. J Clin Hypertens (Greenwich) 2016;18(2):101-108 [FREE Full text] [doi: 10.1111/jch.12682] [Medline: 26456490]

32. Hallberg I, Ranerup A, Kjellgren K. Supporting the self-management of hypertension: patients' experiences of using a mobile phone-based system. J Hum Hypertens 2016;30(2):141-146 [FREE Full text] [doi: 10.1038/jhh.2015.37] [Medline: 25903164]

33. Hallberg I, Ranerup A, Bengtsson U, Kjellgren K. Experiences, expectations and challenges of an interactive mobile phone-based system to support self-management of hypertension: patients' and professionals' perspectives. Patient Prefer Adherence 2018;12:467-476 [FREE Full text] [doi: 10.2147/PPA.S157658] [Medline: 29643739]

34. McGillicuddy JW, Gregoski MJ, Weiland AK, Rock RA, Brunner-Jackson BM, Patel SK, et al. Mobile health medication adherence and blood pressure control in renal transplant recipients: a proof-of-concept randomized controlled trial. JMIR Res Protoc 2013;2(2):e32 [FREE Full text] [doi: 10.2196/resprot.2633] [Medline: 24004517]

35. Persell SD, Karmali KN, Stein N, Li J, Peprah YA, Lipiszko D, et al. Design of a randomized controlled trial comparing a mobile phone-based hypertension health coaching application to home blood pressure monitoring alone: the smart hypertension control study. Contemp Clin Trials 2018;73:92-97. [doi: 10.1016/j.cct.2018.08.013] [Medline: 30172038]

36. Persell SD, Peprah YA, Lipiszko D, Lee JY, Li JJ, Ciolino JD, et al. Effect of home blood pressure monitoring via a smartphone hypertension coaching application or tracking application on adults with uncontrolled hypertension: a randomized clinical trial. JAMA Netw Open 2020;3(3):e200255 [FREE Full text] [doi: 10.1001/jamanetworkopen.2020.0255] [Medline: 32119093]

37. Moore JO, Marshall MA, Judge DC, Moss FH, Gilroy SJ, Crocker JB, et al. Technology-supported apprenticeship in the management of hypertension: a randomized controlled trial. J Clin Outcomes Manag 2014;21(3):110-122.

38. Chandler J, Sox L, Kellam K, Feder L, Nemeth L, Treiber F. Impact of a culturally tailored mHealth medication regimen self-management program upon blood pressure among hypertensive Hispanic adults. Int J Environ Res Public Health 2019;16(7):1226 [FREE Full text] [doi: 10.3390/ijerph16071226] [Medline: 30959858]

39. Davidson TM, McGillicuddy J, Mueller M, Brunner-Jackson B, Favella A, Anderson A, et al. Evaluation of an mHealth medication regimen self-management program for African American and Hispanic uncontrolled hypertensives. J Pers Med 2015;5(4):389-405 [FREE Full text] [doi: 10.3390/jpm5040389] [Medline: 26593951]

40. Table 8.5.d: Criteria for judging risk of bias in the 'Risk of bias' assessment tool. The Cochrane collaboration's tool for assessing risk of bias. 2011. URL: https://handbook-5-1.cochrane.org/chapter 8/ table_8_5_d_criteria_for_judging_risk_of_bias_in_the_risk_of.htm [accessed 2021-07-10]

41. Study quality assessment tools. National Institutes of Health. 2021. URL: https://www.nhlbi.nih.gov/health-topics/ study-quality-assessment-tools [accessed 2021-07-10]

42. Critical appraisal skills programme qualitative checklist. Critical Appraisal Skills Programme. 2018. URL: https://casp-uk. net/wp-content/uploads/2018/01/CASP-Qualitative-Checklist-2018.pdf [accessed 2021-07-10]

43. Duan H, Wang Z, Ji Y, Ma L, Liu F, Chi M, et al. Using goal-directed design to create a mobile health app to improve patient compliance with hypertension self-management: development and deployment. JMIR Mhealth Uhealth 2020;8(2):e14466 [FREE Full text] [doi: 10.2196/14466] [Medline: 32130161$]$

44. Gong K, Yan YL, Li Y, Du J, Wang J, Han Y, APP Study Group. Mobile health applications for the management of primary hypertension: a multicenter, randomized, controlled trial. Medicine (Baltimore) 2020;99(16):e19715 [FREE Full text] [doi: 10.1097/MD.0000000000019715] [Medline: 32311957] 
45. Márquez Contreras E, Márquez Rivero S, Rodríguez García E, López-García-Ramos L, Carlos Pastoriza Vilas J, Baldonedo Suárez A, Compliance Group of Spanish Society of Hypertension (SEH-LELHA). Specific hypertension smartphone application to improve medication adherence in hypertension: a cluster-randomized trial. Curr Med Res Opin 2019;35(1):167-173. [doi: 10.1080/03007995.2018.1549026] [Medline: 30431384]

46. Petrella RJ, Stuckey MI, Shapiro S, Gill DP. Mobile health, exercise and metabolic risk: a randomized controlled trial. BMC Public Health 2014;14:1082 [FREE Full text] [doi: 10.1186/1471-2458-14-1082] [Medline: 25326074]

47. Ovbiagele B, Jenkins C, Patel S, Brunner-Jackson B, Anderson A, Saulson R, et al. Mobile health medication adherence and blood pressure control in recent stroke patients. J Neurol Sci 2015;358(1-2):535-537. [doi: 10.1016/j.jns.2015.10.008] [Medline: 26463572]

48. Kaplan AL, Cohen ER, Zimlichman E. Improving patient engagement in self-measured blood pressure monitoring using a mobile health technology. Health Inf Sci Syst 2017;5(1):4 [FREE Full text] [doi: 10.1007/s13755-017-0026-9] [Medline: 29081974]

49. Ciemins EL, Arora A, Coombs NC, Holloway B, Mullette EJ, Garland R, et al. Improving blood pressure control using smart technology. Telemed J E Health 2018;24(3):222-228. [doi: 10.1089/tmj.2017.0028] [Medline: 28930497]

50. Toro-Ramos T, Kim Y, Wood M, Rajda J, Niejadlik K, Honcz J, et al. Efficacy of a mobile hypertension prevention delivery platform with human coaching. J Hum Hypertens 2017;31(12):795-800. [doi: 10.1038/jhh.2017.69] [Medline: 28972573]

51. Patel S, Jacobus-Kantor L, Marshall L, Ritchie C, Kaplinski M, Khurana PS, et al. Mobilizing your medications: an automated medication reminder application for mobile phones and hypertension medication adherence in a high-risk urban population. J Diabetes Sci Technol 2013;7(3):630-639 [FREE Full text] [doi: 10.1177/193229681300700307] [Medline: 23759395]

52. Weerahandi H, Paul S, Quintiliani LM, Chokshi S, Mann DM. A mobile health coaching intervention for controlling hypertension: single-arm pilot pre-post study. JMIR Form Res 2020;4(5):e13989 [FREE Full text] [doi: 10.2196/13989] [Medline: 32379049]

53. Sharek D, Wiebe E. Measuring video game engagement through the cognitive and affective dimensions. Simul Gaming 2014;45(4-5):569-592. [doi: 10.1177/1046878114554176]

54. Bandura A. Self-efficacy: the exercise of control. New York, NY: W. H. Freeman; 1997.

55. Yeager CM, Benight CC. If we build it, will they come? Issues of engagement with digital health interventions for trauma recovery. Mhealth 2018;4:37 [FREE Full text] [doi: 10.21037/mhealth.2018.08.04] [Medline: 30363749]

56. Adu MD, Malabu UH, Callander EJ, Malau-Aduli AE, Malau-Aduli BS. Considerations for the development of mobile phone apps to support diabetes self-management: systematic review. JMIR Mhealth Uhealth 2018;6(6):e10115 [FREE Full text] [doi: 10.2196/10115] [Medline: 29929949]

57. Sezgin E, Özkan-Yildirim S, Yildirim S. Investigation of physicians' awareness and use of mHealth apps: a mixed method study. Health Policy Technol 2017;6(3):251-267. [doi: 10.1016/j.hlpt.2017.07.007]

58. Fletcher BR, Hinton L, Hartmann-Boyce J, Roberts NW, Bobrovitz N, McManus RJ. Self-monitoring blood pressure in hypertension, patient and provider perspectives: a systematic review and thematic synthesis. Patient Educ Couns 2016;99(2):210-219. [doi: 10.1016/j.pec.2015.08.026] [Medline: 26341941]

59. Shahin W, Kennedy GA, Stupans I. The impact of personal and cultural beliefs on medication adherence of patients with chronic illnesses: a systematic review. Patient Prefer Adherence 2019;13:1019-1035 [FREE Full text] [doi: 10.2147/PPA.S212046] [Medline: 31303749]

60. Li WW, Stewart AL, Stotts N, Froelicher ES. Cultural factors associated with antihypertensive medication adherence in Chinese immigrants. J Cardiovasc Nurs 2006;21(5):354-362. [doi: 10.1097/00005082-200609000-00005] [Medline: 16966912]

61. Kini V, Ho PM. Interventions to improve medication adherence: a review. JAMA 2018;320(23):2461-2473. [doi: 10.1001/jama.2018.19271] [Medline: $\underline{30561486}$ ]

62. Lagun D, Lalmas M. Understanding user attention and engagement in online news reading. In: Proceedings of the 9th ACM International Conference on Web Search and Data Mining. 2016 Presented at: WSDM '16; February 22-25, 2016; San Francisco, CA p. 113-122. [doi: 10.1145/2835776.2835833]

63. Still CH, Jones LM, Moss KO, Variath M, Wright KD. African American older adults' perceived use of technology for hypertension self-management. Res Gerontol Nurs 2018;11(5):249-256 [FREE Full text] [doi: 10.3928/19404921-20180809-02] [Medline: 30230518]

64. Sieck CJ, Walker DM, Gregory M, Fareed N, Hefner JL. Assessing capacity to engage in healthcare to improve the patient experience through health information technology. Patient Exp J 2019;6(2):28-34. [doi: 10.35680/2372-0247.1366]

65. Delva S, Waligora Mendez KJ, Cajita M, Koirala B, Shan R, Wongvibulsin S, et al. Efficacy of mobile health for self-management of cardiometabolic risk factors: a theory-guided systematic review. J Cardiovasc Nurs 2021;36(1):34-55 [FREE Full text] [doi: 10.1097/JCN.0000000000000659] [Medline: 32040072]

66. Norman CD, Skinner HA. eHealth literacy: essential skills for consumer health in a networked world. J Med Internet Res 2006;8(2):e9 [FRE Full text] [doi: 10.2196/jmir.8.2.e9] [Medline: 16867972] 


\section{Abbreviations}

BP: blood pressure

mHealth: mobile health

NIH: National Institutes of Health

PRISMA: Preferred Reporting Items for Systematic Reviews and Meta-Analyses

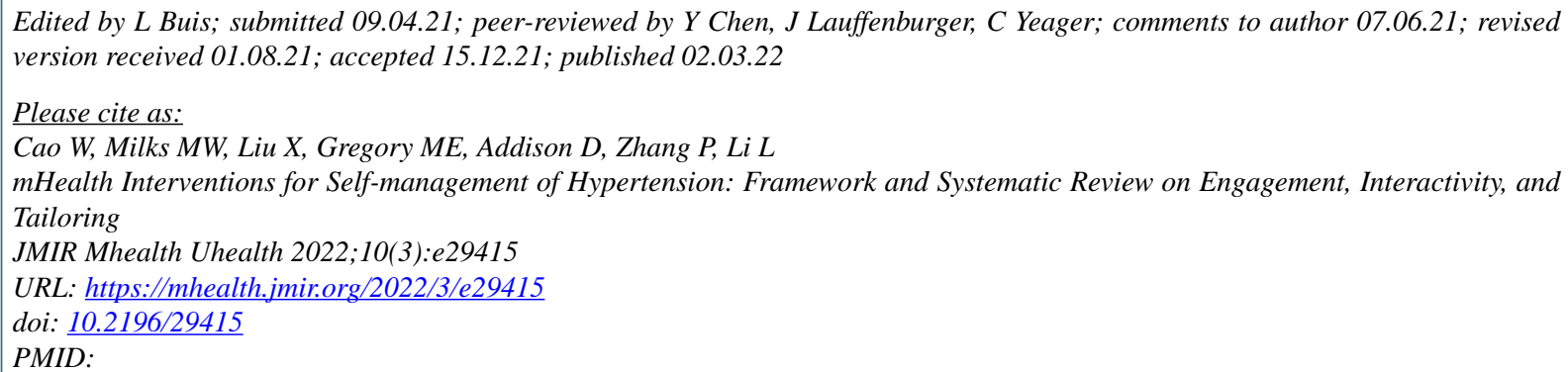

(CWeidan Cao, M Wesley Milks, Xiaofu Liu, Megan E Gregory, Daniel Addison, Ping Zhang, Lang Li. Originally published in JMIR mHealth and uHealth (https://mhealth.jmir.org), 02.03.2022. This is an open-access article distributed under the terms of the Creative Commons Attribution License (https://creativecommons.org/licenses/by/4.0/), which permits unrestricted use, distribution, and reproduction in any medium, provided the original work, first published in JMIR mHealth and uHealth, is properly cited. The complete bibliographic information, a link to the original publication on https://mhealth.jmir.org/, as well as this copyright and license information must be included. 